\title{
Atrial Fibrillation Driver Localization From Body Surface Potentials Using Deep Learning
}

\author{
Miguel Ángel Cámara-Vázquez ${ }^{a}$, Adrián Oter-Astillero ${ }^{a}$, Ismael Hernández-Romero ${ }^{a}$, Miguel \\ Rodrigo $^{b}$, Eduardo Morgado-Reyes ${ }^{a}$, María S. Guillem ${ }^{b}$, Andreu M. Climent ${ }^{b}$, Óscar Barquero-Pérez $^{a}$ \\ ${ }^{a}$ Universidad Rey Juan Carlos, Fuenlabrada, Madrid, Spain \\ ${ }^{b}$ ITACA Institute, Universitat Politècnica de València, València, Spain
}

\begin{abstract}
Atrial fibrillation $(A F)$ is characterized by complex and irregular propagation patterns. Multipoint intracardiac mapping systems present a limited spatial resolution, which makes it difficult to identify $A F$ drivers and ablation targets. These $A F$ onset locations and drivers responsible for $A F$ perpetuation are main targets for ablation procedures. Although noninvasive electrocardiographic imaging (ECGI) and inverse problem-based methods have been tested during AF conditions, they need an accurate mathematical modeling of atria and torso to get good results. In this work, we propose to model the location of AF drivers from body surface potentials (BPS) as a supervised classification problem. We used deep learning techniques to address the problem. We were able to correctly locate the $92 \%$ and $96 \%$ of drivers in the test and training sets, respectively (accuracy of 0.92 and 0.96), while the Cohen's Kappa was 0.89 for both sets. Therefore, proposed method can help to identify target regions for ablation using a noninvasive procedure as BSP mapping.
\end{abstract}

\section{Introduction}

Atrial fibrillation (AF) is the most common type of arrhythmia in clinical practice. AF affects more than 33 million patients in the world [1]. Patients with this condition have an increased risk of suffering embolism, cardiac failure, stroke and, in the worst of cases, death [2]. One of the clinical goals in $\mathrm{AF}$ patients is to restore sinus rhythm, usually by ablation. Main targets of ablation are AF onset locations and drivers responsible for AF perpetuation [3]. Several studies have proposed different strategies to locate these AF drivers by applying the inverse problem of ECG Imaging (ECGI) with promising results [4-7]. However, the inverse problem approach still needs further improvement. We propose to model the location of AF drivers from body surface potentials (BPS) as a supervised classification problem. We used deep learning techniques, as a su- pervised model, to address the location of AF drivers from conveniently annotated realistic computerized AF models [8].

The use of Deep Learning (DL) techniques have undergone considerable development in bioengineering the last decade. DL has been used in AF detection by using RNNs and CNNs [9], by STFT, stationary wavelet transform and CNNs [10], and in the detection of individuals at risk of suffering from Paroxysmal AF by CNNs [11].

The remaining of the paper is organized as follows. In Section 2 we introduce the computational models used for this study, the experimental set-up, performance metrics and Deep Learning architecture. Final results are summarized in Section 3 and in Section 4 main conclusions are presented.

\section{Methods}

\subsection{Computerized Models}

We used realistic computerized models of atria $(\mathrm{N}=2039$ nodes) and torso (M=659 nodes) $[6,8,12]$. This geometrical model considers a simplified unique endocardiumepicardium layer for the atrial tissue. Atria and torso models were used to simulate 13 different AF propagation patterns in both left atria (LA) and right atria (RA), with different complexity and driver positions: Posterior Left Atrial Wall (PLAW), Left Inferior Pulmonary Vein (LIPV), Left Superior Pulmonary Vein (LSPV), Right Inferior Pulmonary Vein (RIPV), Right Superior Pulmonary Vein (RSPV), Right Atrial Appendage (RAA) and Right Atria Free Wall (RAFW).

\subsection{Modeling AF driver location as a clas- sification problem}

We proposed to address the location of AF drivers as a supervised classification problem. We divided the atria into 7 regions (Figure 1-a) where the AF drive can be localized [13]. Each region represent a class to which the 
a)

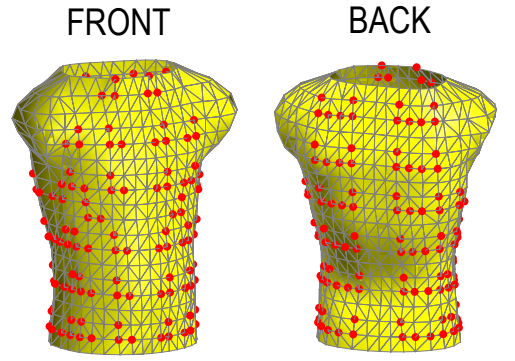

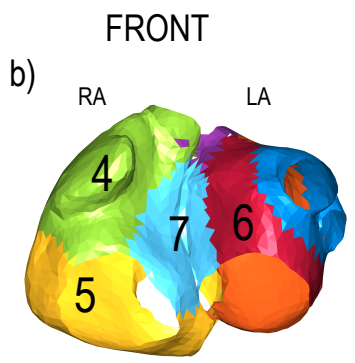

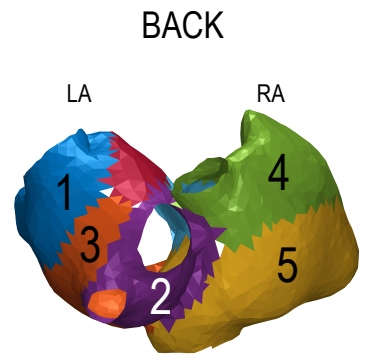

Figure 1. a) Selected BSP in torso and back. b) Regions in atria where AF drivers are found.

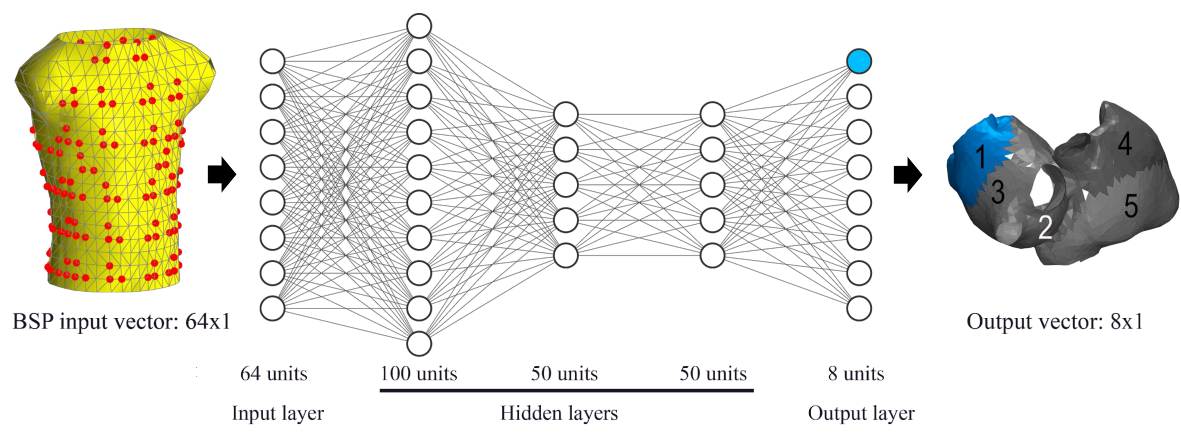

Figure 2. Schematic overview of the Multilayer Perceptron (MLP)-based proposed method.

AF driver belongs. To obtain labeled data, AF driver location from the computerized model were manually classified into one region for each time-instant.

To obtain the input data (simulated BSP), the forward problem of ECGI was solved by computing the $M \times N$ transfer matrix A using the boundary element method [6, $14,15]$. Simulated BSP were referenced to the Wilson Terminal Center, corrupted with additive Gaussian noise $(\mathrm{SNR}=20 \mathrm{dBs})$ and filtered using a $4^{\text {th }}$-order bandpass Butterworth filter ( $\mathrm{fc}_{1}=3 \mathrm{~Hz}$ and $\left.\mathrm{fc}_{2}=30 \mathrm{~Hz}\right)[6,8]$.

A set of 64 electrodes from the whole torso geometry were selected to represent realistic multi-electrode vest used in electrophysiological studies, see Figure 1-a).

\subsection{Deep-Learning Architecture}

One typical Artificial Neural Network (ANN) is the Multilayer Perceptron (MLP). It consists on a series of groups of neurons (layers) that are connected between them. Each neuron applies an activation function for the weighted sum of its inputs, and the result is transmitted to the next neuron. This type of non-linear model is inspired by the neural architecture of the brain [16].

For this study we trained an MLP with 3 hidden layers $(100,50,50)$ units each. The output layer consists on 8 units, one per region, and one for no AF driver. We used ReLu activation function in hidden layers and Softmax in the output. This Softmax layer allows to obtain the probability of the input sample to belong to each region. A schematic overview of this model can be seen in Figure 2.

\subsection{Performance metrics}

To assess the performance of the implemented DL models, we used two different metrics. The first one is the accuracy (Acc), measured as the fraction of well-classified drivers (true positives, TP) over the total of drivers (Total):

$$
A c c=\frac{T P}{\text { Total }}
$$

The second performance metric is the Cohen's kappa. It is a robust statistic used for rating reliability testing [17]. A score of 0 means the agreement that can be expected from random chance, whereas a score of 1 represents perfect agreement between the raters. Scores less than 0 means that there is less agreement than chance. It is computed as:

$$
\kappa=\frac{p_{o}-p_{e}}{1-p_{e}}
$$

where $p_{o}$ is the relative agreement among raters (identical to accuracy), and $p_{e}$ the hypothetical probability of chance agreement.

\subsection{Experimental set-up}

We first addressed the problem considering each time instant independently. So that, the whole data set is comprised of input data vectors with 64 entries from the BSP 
Table 1. Obtained accuracy and Cohen's kappa for independent time instants and model independence.

\begin{tabular}{lcccc}
\hline & Train Acc & Test Acc & Train Kappa & Test Kappa \\
\hline Independent time instants & 0.955 & 0.912 & 0.893 & 0.893 \\
Independent time instants (CW) & 0.961 & 0.925 & 0.895 & 0.895 \\
Model independence & 0.823 & 0.452 & 0.724 & 0.723 \\
Model independence (CW) & 0.815 & 0.439 & 0.727 & 0.725 \\
\hline
\end{tabular}
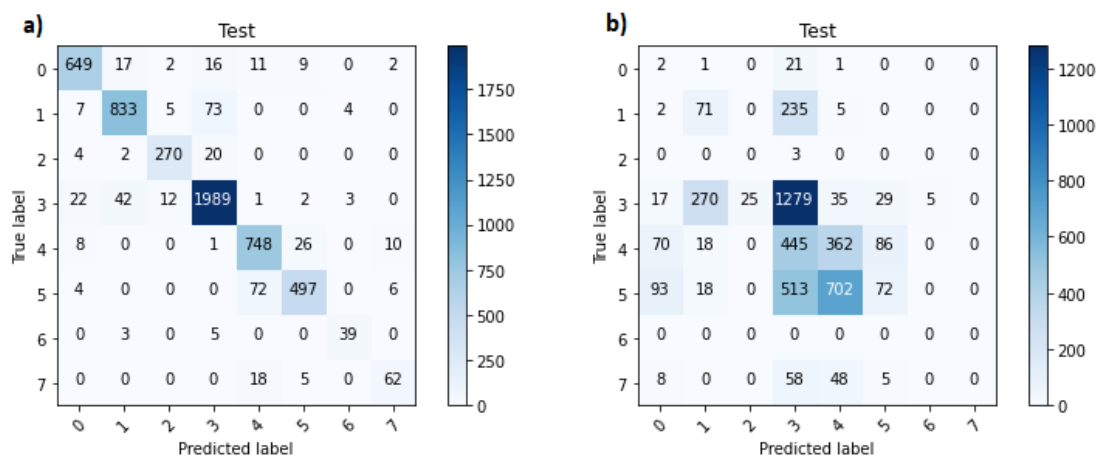

Figure 3. Confusion matrices obtained for independent time instants (a) and model independence methods (b).

electrodes, $\boldsymbol{x}_{\boldsymbol{i}}$, and the corresponding label $y_{i}$ which can have values $0,1, \ldots, 7$. The whole data set was split into training $(80 \%)$ and test $(20 \%)$ sets. This approach allowed samples from the same model to be in the training and in the test set. Secondly, to avoid the bias introduced by the first approach, we consider model independence, thus samples from a model are not allowed to be simultaneously in training and test set.

The problem is imbalanced, since there are several atria regions which are over represented in the data set. In order to address this problem we tried to weigh the classes accordingly to the probability of occurrence.

\section{Results}

Table 1 shows the results considering each time instant as an independent sample, thus allowing samples from the same model to be in the training and in the test set. Table 1 shows also results considering independence by models, where samples from a given model cannot be simultaneously in training and test sets. With this approach, we were able to correctly locate the $95 \%$ and $91 \%$ of drivers in the training and test sets, respectively (accuracy of 0.95 and 0.91), while the Cohen's Kappa was 0.893 for both sets.

Using class weight (CW) to fight imbalance we slightly improved the metrics, being able to correctly locate the $96 \%$ and $92 \%$ of drivers in the training and test sets, respectively, while the Cohen's Kappa was 0.895 for both sets.

In Figure 3-a the confusion matrix for this approach (test set, CW) is shown. In the case of the label 3, the $96 \%$ of drivers are well classified, but this percentage drops to the $77 \%$ in the case of the label 7, with much less data available.

Table 1 shows results for the approach of splitting the data set by Models (using also class weight). This is a more realistic and fair scenario. The accuracy for the training set are 0.82 and 0.81 using CW (0.72 for Cohen's Kappa), while accuracy for the test set was 0.45 and 0.44 using $\mathrm{CW}$. These results suggest that we are overfitting the model to the training set. However, Cohen's Kappa maintains a value of 0.72 , which indicated that the model is able to fight the imbalance problem.

In Figure 3-b the confusion matrix for this approach (test set, $\mathrm{CW}$ ) is shown.

\section{Discussion and conclusions}

The deep learning method we proposed can help to identify target regions for ablation using a non-invasive procedure, as BSP mapping. The main advantage of this methodology is to predict those regions without using ECGI, which requires accurate mathematical modeling of torso and atria.

Although the results we got are very promising, there are some drawbacks that should be addressed. The main problem we had to face is the imbalance of input data. Our dataset is composed by only 13 models that represent different propagation patterns, but the distribution of drivers across the 7 defined atrial regions is not balanced. Therefore, the results on those less represented regions are going to be worse. It also can lead to overfitting to the training set (problem that can be shown in the second proposed model). One way to solve this problem is to test 
our methodology with a higher number of propagation patterns. Overfitting to the training set can be also fixed using dropout, regularization or data augmentation. For example, data augmentation can be achieved by training the models with signals with different SNRs.

Finally, future work is to apply this methodology with real patient data, where driver tagging can be difficult. For this task, Convolutional Neural Networks could be useful, since they can use the spatial characteristics of BSP that are omitted when using MLP.

\section{Acknowledgements}

This work has been partially supported by projects TEC2016-75361-R and PID2019-105032GB-I00 from the Spanish Ministry of Economy and Spanish Ministry of Science and Innovation, respectively, projects IJCI-2014-22178, PI16-01123, DTS16/00160, PI17/0159, PI17/01106 from the Spanish Ministry of Economy through the Carlos III Health Institute with FEDER founds, grant GVA APOSTD/2017/068 and projects AICO/2018/267, GV/2018/103 from the Education, Research, Culture and Sports department of Generalitat Valenciana.

\section{References}

[1] Chugh SS, Havmoeller R, Narayanan K, Singh D, Rienstra M, Benjamin EJ, Gillum RF, Kim YH, McAnulty JH, Zheng ZJ, Forouzanfar MH, Naghavi M, Mensah GA, Ezzati M, Murray CJ. Worldwide epidemiology of atrial fibrillation: A global burden of disease 2010 study. Circulation 2014;129(8):837-847. ISSN 00097322.

[2] Fuster V, Rydén LE, Cannom DS, Crijns HJ, Curtis AB, Ellenbogen KA, Halperin JL. Acc/aha/esc 2006 guidelines for the management of patients with atrial fibrillation: full text. Europace 2006;8(9):651-745.

[3] Guillem M, Climent A, Rodrigo M, Fernández-Avilés F, Atienza F, Berenfeld O. Presence and stability of rotors in atrial fibrillation: evidence and therapeutic implications. Cardiovascular Res 2016;109(4):480-492.

[4] Haissaguerre M, Hocini M, Shah AJ, Derval N, Sacher F, Jais P, Dubois R. Noninvasive panoramic mapping of human atrial fibrillation mechanisms: A feasibility report. Journal of Cardiovascular Electrophysiology 2013; 24(6):711-717.

[5] Rodrigo M, Climent AM, Liberos A, Calvo D, FernándezAvilés F, Berenfeld $\mathrm{O}$, et al. Identification of dominant excitation patterns and sources of atrial fibrillation by causality analysis. Annals of Biomedical Engineering 2016; 44(8):2364-2376.

[6] Pedrón-Torrecilla J, Rodrigo M, Climent A, Liberos A, Pérez-David E, Bermejo J, et al. Noninvasive estimation of epicardial dominant high-frequency regions during atrial fibrillation. J Cardiovascular Electrophysiol 2016; 27(4):435-442.
[7] Cuculich PS, Wang Y, Lindsay BD, Faddis MN, Schuessler RB, Damiano RJ, Li L, Rudy Y. Noninvasive characterization of epicardial activation in humans with diverse atrial fibrillation patterns. Circulation 2010;122(14):1364-1372.

[8] Figuera C, Suárez-Gutiérrez V, Hernández-Romero I, Rodrigo M, Liberos A, Atienza F, Guillem MS, BarqueroPérez Ó, Climent AM, Alonso-Atienza F. Regularization Techniques for ECG Imaging during Atrial Fibrillation: A Computational Study. Frontiers in Physiology oct 2016; 7:466. ISSN 1664-042X.

[9] Xiong Z, Stiles M, Zhao J. Robust ECG Signal Classification for the Detection of Atrial Fibrillation Using Novel Neural Networks. September 2017; .

[10] Xia Y, Wulan N, Wang K, Zhang H. Detecting atrial fibrillation by deep convolutional neural networks. Computers in Biology and Medicine February 2018;93:84-92. ISSN 00104825.

[11] Pourbabaee B, Roshtkhari MJ, Khorasani K. Deep Convolutional Neural Networks and Learning ECG Features for Screening Paroxysmal Atrial Fibrillation Patients. IEEE Transactions on Systems Man and Cybernetics Systems December 2018;48(12):2095-2104. ISSN 2168-2216, 21682232.

[12] García-Molla V, Liberos A, Vidal A, Guillem M, Millet J, González A, et al. Adaptive step $\{\mathrm{ODE}\}$ algorithms for the $3 \mathrm{~d}$ simulation of electric heart activity with graphics processing units. Computers in Biology and Medicine 2014; $44: 15-26$.

[13] Haissaguerre M, Hocini M, Denis A, Shah AJ, Komatsu Y, Yamashita S, et al. Driver domains in persistent atrial fibrillation. Circulation 2014;130(7):530-538.

[14] Barr RC, Ramsey M, Spach MS. Relating epicardial to body surface potential distributions by means of transfer coefficients based on geometry measurements. IEEE Transactions on biomedical engineering 1977;(1):1-11.

[15] De Munck J. A linear discretization of the volume conductor boundary integral equation using analytically integrated elements. IEEE Transactions on Biomedical Engineering 1992;39(9):986-990.

[16] Castro W, Oblitas J, Santa-Cruz R, Avila-George H. Multilayer perceptron architecture optimization using parallel computing techniques. PLOS ONE December 2017; 12(12):e0189369. ISSN 1932-6203. Publisher: Public Library of Science.

[17] McHugh ML. Interrater reliability: the kappa statistic. Biochemia Medica October 2012;22(3):276-282. ISSN 13300962.

Address for correspondence:

Óscar Barquero-Pérez (oscar.barquero@urjc.es). Dept. IIID207, Camino del Molino, 5. 28943 - Fuenlabrada (Madrid), Spain. 